УДК 004.02:537.86:621.391.8

\title{
ОПРЕДЕЛЕНИЕ АМПЛИТУДНЫХ УРОВНЕЙ КУСОЧНО-ПОСТОЯННОГО СИГНАЛА ПОСРЕДСТВОМ ПОЛИНОМИАЛЬНОЙ АППРОКСИМАЦИИ
}

\author{
С. М. ВОВК, В. Ф. БОРУЛЬКО \\ Днепропетровский национальный университет им. О. Т. Гончара, \\ Украина, Днепропетровск, 49050, ул. Научная, 13
}

\begin{abstract}
Аннотация. Предложен новый подход к определению амплитудных уровней кусочно-постоянного сигнала, который основан на использовании его мультипликативной модели и решении задачи полиномиальной аппроксимации. Для случая отсутствия шума постановка задачи полиномиальной аппроксимации основана на требовании точного совпадения текущего значения сигнала со значением амплитуды одного из его уровней. Для случая наличия обычного аддитивного шума постановка задачи основана на критерии наименьших квадратов, а решение представлено в аналитическом виде. Для случая наличия шума импульсного типа постановка задачи основана на критерии минимума длительности, а решение достигается численно путем минимизации соответствующего функционала по неизвестным амплитудам уровней. Подробно рассмотрен случай бинарного кусочно-постоянного сигнала. Приведены результаты числового моделирования для случаев, когда бинарный сигнал искажен обычным аддитивным шумом с законом распределения Гаусса и шумом импульсного типа с законом распределения Коши.
\end{abstract}

Ключевые слова: кусочно-постоянный сигнал; уровень амплитуды; скачок амплитуды; полиномиальная аппроксимация; стоимостная функция; метод минимума длительности

\section{ВВЕДЕНИЕ}

Модель кусочно-постоянного сигнала (КПС) является одной из важных и полезных моделей в области обработки сигналов и изображений [1-5]. Обычно полагают, что КПС состоит из достаточно протяженных участков постоянной амплитуды - амплитудных уровней, переключения между которыми выполняются мгновенно. В связи с этим математическая модель КПС традиционно задается «кусками» (сегментами), где каждый сегмент сигнала характеризуется своей амплитудой и моментом времени переключения на эту амплитуду. Задача восстановления КПС состоит в определении значений амплитуд его уровней и моментов времени их переключения. Если влиянием системы, которая формирует или ре- гистрирует КПС, возможно пренебречь, а шумом пренебречь нельзя, то задача восстановления КПС формулируется как задача подавления шума [6].

Важным частным случаем задачи восстановления КПС является задача определения амплитуд его уровней. Классический подход к решению этой задачи основан на кластеризации значений КПС [3]. Но кластеризация, выполняемая на базе традиционных алгоритмов K-means или K-medians [7], является адекватным подходом только для случая обычного аддитивного шума с законами распределения Гаусса или Лапласа, соответственно. Поэтому она приводит к сдвигу центров кластеров тогда, когда сигнал искажен шумом импульсного типа, например, шумом Коши. При этом отметим, что алгоритм K-medians, запускаясь из не- 


\section{БИБЛИОГРАФИЧЕСКИЙ СПИСОК}

1. Parekh, A.; Selesnick, I. W. Convex fused lasso denoising with non-convex regularization and its use for pulse detection. Proc. of IEEE Signal Processing in Medicine and Biology Symp., Philadelphia, PA. IEEE, 2015. - P. 1-6. - DOI : 10.1109/SPMB.2015.7405474.

2. Zhang, Kaihua; Liu, Qingshan; Song, Huihui; Li, Xuelong. A variational approach to simultaneous image segmentation and bias correction. IEEE Trans. Cybernetics. - Aug. 2015. - Vol. 45, No. 8. - P. 1426-1437. - DOI : $10.1109 /$ TCYB.2014.2352343.

3. Little, Max A.; Jones, Nick S. Generalized methods and solvers for noise removal from piecewise constant signals. I. Background theory. Proc. R. Soc. A. 2011. - Vol. 467. - P. 3088-3114. - DOI : 10.1098/rspa.2010.0671.

4. Little, Max A.; Jones, Nick S. Generalized methods and solvers for noise removal from piecewise constant signals. II. New methods. Proc. R. Soc. A. 2011. - Vol. 467. - P. 3115-3140. - DOI : 10.1098/rspa.2010.0674

5. Ongie, Greg; Jacob, Mathews. Recovery of piecewise smooth images from few Fourier samples. Proc. of Int. Conf. on Sampling Theory and Applications, SampTA 25-29 May 2015. - IEEE, 2015. - P. 543-547. — DOI : $10.1109 /$ SAMPTA.2015.7148950.

6. Selesnick, I. W.; Figueiredo, M. A. T. Signal restoration with overcomplete wavelet transforms: comparison of analysis and synthesis priors. Proc. SPIE. - 2009. - Vol. 7446 (Wavelets XIII). - P. 74460D-74460D-15. - DOI : 10.1117/12.826663.

7. Aggarwal, C. C.; Reddy C. K. Data clustering: algorithms and applications, N.-Y.: Chapman and Hall/CRC, 2013. - 652 p.

8. Вовк, С.М.; Борулько, В.Ф.Метод минимума длительности для восстановления финитных сигналов. Известия вузов. Радиоэлектроника. — 1991. - Т. 34, № 8. - C. 66-69.

9. Borulko, V. F.; Vovk, S. M. Minimum-duration filtering. Радиоэлектроника. Информатика. Управление. - 2016. - № 1. - C. 7-14. - DOI : 10.15588/1607-3274-2016-1-1.

10. Gonzalez, J. G.; Arce, G. R. Optimality of the myriad filter in practical impulsive-noise environments. IEEE Trans. Signal Process. - Feb. 2001. - Vol. 49, No. 2. - P. 438-441. - DOI : 10.1109/78.902126.

11. Aysal, T. C.; Barner, K. E. Meridian filtering for robust signal processing. IEEE Trans. Signal Process. - 
Aug. 2007. - Vol. 55, No. 8. - P. 3949-3962. — DOI : 10.1109/TSP.2007.894383.

12. Carrillo, R. E.; Aysal, T. C.; Barner, K. E. Generalized Cauchy distribution based robust estimation. Proc. of Int. Conf. on Acoustic, Speech and Signal Processing, ICASSP, 31 Mar.-4 Apr. 2008, Las Vegas. IEEE, 2008. - P. 3389-3392. - DOI : 10.1109/ICASSP.2008.4518378.

13. Вовк, С. М.; Борулько, В. Ф. Постановка задач определения линейных параметров сигналов в квазинормированных пространствах. Известия вузов. $\mathrm{Pa}$ диоэлектроника. — 2010. — Т. 53, № 7. — С. 31-42. -
URL

http://radio.kpi.ua/article/view/S0021347010070046.

14. Borulko, V. F.; Vovk, S. M. Principle of minimum extent in spatial spectrum extrapolation problems of complex-valued sources. Telecom. Radio Eng. - 2013. - Vol. 72, No. 7. - P. 581-592. - DOI : $\underline{10.1615 / \text { TelecomRadEng.v72.17.30. }}$

15. Вовк, С.М.; Борулько, В.Ф.Двойственный метод минимума пространственной протяженности для робастного оценивания параметров дипольных источников излучения. Радиоэлектроника. Информатика. Управление. — 2014. — № 2. - C. 8-17. — DOI : 10.15588/1607-3274-2014-2-1. 